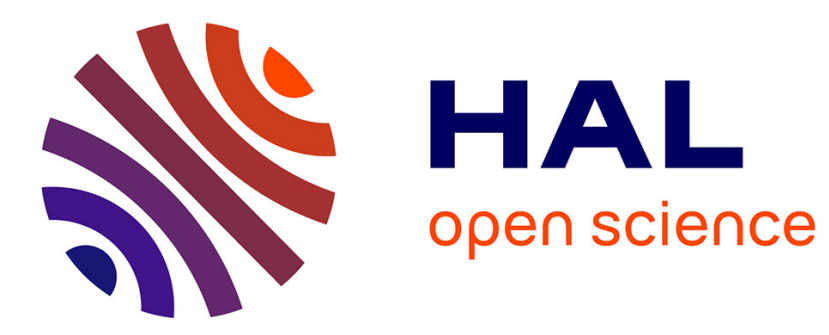

\title{
Multi(ples) appartenances en milieu rural
}

Yannick Sencébé

\section{- To cite this version:}

Yannick Sencébé. Multi(ples) appartenances en milieu rural. Informations sociales, 2011, 164, pp.3642. hal-02643960

\section{HAL Id: hal-02643960 \\ https://hal.inrae.fr/hal-02643960}

Submitted on 28 May 2020

HAL is a multi-disciplinary open access archive for the deposit and dissemination of scientific research documents, whether they are published or not. The documents may come from teaching and research institutions in France or abroad, or from public or private research centers.
L'archive ouverte pluridisciplinaire HAL, est destinée au dépôt et à la diffusion de documents scientifiques de niveau recherche, publiés ou non, émanant des établissements d'enseignement et de recherche français ou étrangers, des laboratoires publics ou privés. 


\title{
MULTI(PLES) APPARTENANCES EN MILIEU RURAL
}

\author{
Yannick Sencébé
}

CNAF | Informations sociales

\section{$2011 / 2-n^{\circ} 164$ \\ pages 36 à 42}

\section{ISSN 0046-9459}

Article disponible en ligne à l'adresse:

http://www.cairn.info/revue-informations-sociales-2011-2-page-36.htm

Pour citer cet article :

Sencébé Yannick, «Multi(ples) appartenances en milieu rural »,

Informations sociales, $2011 / 2 n^{\circ} 164$, p. 36-42.

Distribution électronique Cairn.info pour CNAF.

(c) CNAF. Tous droits réservés pour tous pays.

La reproduction ou représentation de cet article, notamment par photocopie, n'est autorisée que dans les limites des conditions générales d'utilisation du site ou, le cas échéant, des conditions générales de la licence souscrite par votre établissement. Toute autre reproduction ou représentation, en tout ou partie, sous quelque forme et de quelque manière que ce soit, est interdite sauf accord préalable et écrit de l'éditeur, en dehors des cas prévus par la législation en vigueur en France. II est précisé que son stockage dans une base de données est également interdit. 


\section{Multi(ples) appartenances en milieu rural}

Yannick Sencébé - sociologue

Depuis les années 1970, on constate une certaine attractivité des campagnes; aujourd'hui, des populations aux profils sociaux hétérogènes cohabitent en milieu rural. Quelles sont, entre attachement et distanciation, leurs différentes formes d'appartenance aux lieux, et quels liens tissent-elles entre elles?

Le monde rural a longtemps été, et demeure dans l'imaginaire collectif, associé aux racines, à l'autochtonie et à la succession des générations dans un même lieu. Cette image, associée aux valeurs d'authenticité et de convivialité, est concomitante depuis les années 1970 à un bouleversement total de la sociologie des campagnes. L'exode rural, qui a vidé les campagnes des trois quarts de leur population et y a fixé au sol ceux qui n'ont pas pu ou voulu prendre le chemin des villes et de la modernisation, se renverse en effet à partir de 1975, d'après les chiffres du recensement de population. La crise économique, les limites d'une ville qui donne ses premiers symptômes de saturation, l'anonymat qui règne dans les grands ensembles commencent à produire leurs effets. La campagne va redevenir attractive pour de multiples raisons. Lieu préservé de la société de consommation où il serait possible de vivre une utopie politique à travers le «retour à la terre » et l'expérience communautaire, telle est la vision de la génération de mai 1968. Lieu de retrouvailles familiales où il est possible de célébrer la mémoire généalogique, telle est la destinée des régions à fort taux de résidence secondaire. Lieu où, à défaut de pouvoir changer la vie, il est possible de changer de vie, tel est le crédo des néoruraux qui, à partir des années 1980, vont tenter leur chance, "prendre un nouveau départ » en créant leur propre emploi ou en bricolant dans l'économie informelle et l'entraide des réseaux «d'anciens néo ». Lieu, enfin, où il est possible d'acquérir à moindre coût une maison individuelle 
supposée favoriser l'épanouissement familial, tel est aujourd'hui le moteur de la périurbanisation qui s'étend toujours plus loin dans le rural "profond ». Cette liste non exhaustive laisse apparaître l’hétérogénéité des profils sociaux présents dans les campagnes et des manières de les habiter.

Cet article propose une lecture des formes plurielles d'appartenance en milieu rural, c'est-à-dire des manières dont les individus sont inscrits dans des lieux, et des façons dont ils tissent et entretiennent leurs relations sociales. Ainsi, nous parlerons de «formes d'appartenance » plutôt que de «multiappartenance », car cette dernière notion met l'accent sur la mobilité et la diversité des lieux d'inscription des individus, ce qui cache l'importance des phénomènes de sédentarité et d'autochtonie en milieu rural, d'une part, et des inégalités sociales face à la mobilité, d'autre part (Kaufmann, 2005). L'errance ne se confond pas avec le nomadisme, et les populations captives d'un espace ne vivent pas la même réalité que celles qui ont fait le choix de l'ancrage. Cet article de synthèse reprend différents travaux menés en milieu rural (vallée du Diois et commune de Cobonne dans la Drôme, pays de Puisaye-Forterre dans l'Yonne). Les résultats présentés s'appuient sur des entretiens approfondis (récits de vie) avec des habitants rencontrés par réseau, de proche en proche, et choisis pour la diversité de leur rapport à l'espace local et de leur position sociale (paysan autochtone, diplomate en résidence secondaire, néorural, ouvrier venu chercher du travail...). Nous avons ainsi distingué, de manière typologique, quatre formes d'appartenance en tenant compte de deux pôles entre lesquels se positionnent les individus : l'attachement et la distanciation (Sencébé, 2008). L'attachement fait référence à notre besoin d'identification, d'affiliation et de sécurité. La distanciation ramène plus directement aux valeurs de liberté, de projet, d'épanouissement personnel.

\section{L'attachement comme destin social}

Dans la première forme d'appartenance, le pôle de l'attachement est prédominant. Le lieu où l'on vit renferme toutes les sphères d'affiliation. Ce lieu peut prendre la forme du village, du quartier, de la ferme, du pays. L'entretien des liens passe par la proximité et le temps long. Le lieu est parcouru, travaillé, connu en ses moindres parties de façon topologique et généalogique. On est ainsi à la fois partie prenante et dépendant du lieu que l'on construit et qui nous construit, avec d'autres. Dans un monde où prédominait la sédentarité, ce type d'appartenance a caractérisé bon nombre de personnes, si ce n'est la majorité. La civilisation paysanne décrite par Henri Mendras (1977) en est l'illustration. L'interconnaissance au sein d'une localité offrait à ses membres l'essentiel de leurs relations, moyens et raisons d'exister. Autant d'éléments qui ont été considérablement modifiés, y compris dans les campagnes les plus reculées. Ainsi, dans le contexte présent où au contraire prédomine la mobilité, cette forme d'appartenance peut être vécue à travers 
la perte - perte des siens partis ailleurs, perte de repères lorsque change radicalement le lieu où l'on croyait pouvoir perpétuer une façon d'être au monde. L'univers dans lequel on vit est un monde qui apparait aujourd'hui clos et limité au regard de l'aisance avec laquelle d'autres gèrent leurs cheminements et déménagements. De cet univers, pourtant, on tire son identité et sa subsistance, sans pouvoir en retour en contrôler le présent ni l'avenir. C'est, nous semble-t-il, ce qui caractérise les classes populaires rurales, marquées en cela par une crise du «capital d'autochtonie » (1) Rénahy, 2005). Qu'il s'agisse de la fraction prolétarisée des agriculteurs ou des ouvriers des industries encore nombreuses en milieu rural, le destin tracé au fil des générations devient prison ou ghetto en l'absence d'avenir ici (impossibilité de transmettre une exploitation trop petite ou de suivre la course à l'agrandissement, impossibilité de s'insérer ou de se maintenir dans l'emploi quand la seule usine de la région délocalise ou ferme ses portes) et de perspectives ailleurs. Pourtant, certains signes semblent porteurs d'une revalorisation de l'attachement. Le goût immodéré pour le patrimoine, la recherche de racines, les mouvements qui, un peu partout, cherchent à relocaliser la vie et l'économie, tout cela pourrait contribuer à faire apparaître le «paysan» ou le «gars du coin » comme figure de résistance et d'alternative au «nouvel esprit du capitalisme » (Boltanski et Chiapello, 1999). Rappelons que cette idéologie aujourd'hui dominante discrédite les affiliations collectives durables (l'enracinement, la « carrière », la sécurité sociale en général) et valorise au contraire la mobilité, la flexibilité, l'investissement de chacun dans des "projets » sans cesse à renouveler.

\section{L'ancrage comme choix}

«C'est mon choix» est une formule qui caractérise bien le rapport qu'un certain nombre d'habitants (plus ou moins « nouveaux») entretiennent avec la vie à la campagne. Contrairement à l'attachement, l'ancrage en milieu rural n'est pas vécu comme le seul horizon possible : on peut toujours lever l'ancre. Il s'inscrit dans un parcours social et géographique qui a permis de se constituer un certain nombre de ressources (compétences professionnelles, réseaux, intelligence du milieu où l'on se trouve...) au fil des expériences

66 (...) l'ancrage en milieu rural (...) s'inscrit dans un parcours social et géographique (...). 99 accumulées, et de savoir pourquoi on est là. On est ainsi partie prenante du lieu parce qu'on l'a choisi, on s'y engage de façon volontaire tout en gardant la possibilité de s'aérer, de se ressourcer par le fait de pouvoir en sortir (par des réseaux, des visites...). On saisit d'autant mieux la valeur du lieu où l'on vit que l'on peut faire l'expérience de l'«autre», de l'«ailleurs ». Cette forme d'appartenance caractérise une partie des classes moyennes qui réinvestissent, depuis les années 1970, les campagnes. L'apport de ces populations au milieu rural est indéniable. Elles jouent un rôle essen- 
tiel dans la valorisation patrimoniale, touristique, écologique des campagnes. Leur implication dans les conseils municipaux et dans la vie associative et culturelle en fait également des acteurs importants du développement local et du lien social. Mais, selon nous, la prise en compte et la participation des plus «attachés » semble déterminante pour éviter les effets d'exclusion sociale et l'oubli de la mémoire longue, du savoir populaire et de la connaissance fine des lieux que ceux-ci possèdent.

\section{La tension entre l'ici et l'ailleurs}

C'est sans doute ici que l'on pourrait le plus aisément parler de «multiappartenance ». L'attachement au lieu d'origine et l'engagement dans des liens sociaux situés ailleurs (partir faire sa vie, trouver une situation, mener une carrière, fonder un foyer...) caractérisent ce genre de situations de plus en plus communes dans une société valorisant la mobilité. La conscience qu'il n'y a rien à faire ici et que l'on doit chercher à partir, tout en restant d'ici, marque ce cas de figure. Il fut d'abord le fait d'une génération, celle qui a suivi les chemins de l'exode à l'époque où la ville signifiait la promesse d'une ascension sociale et dont une bonne partie a gardé une attache à travers la maison familiale, secondaire puis principale à l'heure de la retraite. Ces carrières résidentielles et sociales ont engendré une tension entre un ici, pourvoyeur de l'identité familiale, et un ailleurs, porteur de l'identité sociale. Comme bon nombre de déracinés, leur rapport au lieu d'origine est ambivalent. Certains acceptent difficilement le changement, le développement, la modernisation de ce lieu de mémoire inscrit dans un passé souvent mythifié. Pour d'autres, la résidence secondaire est le vestibule de l'ancrage sur le mode précédemment décrit. Mais cette forme d’appartenance en se généralisant pourrait changer de sens et n'être qu'un des passages obligés dans les carrières résidentielles qui se construisent dorénavant dans un aller-retour entre ville et campagne selon les cycles de vie.

\section{Le détachement comme injonction sociale}

Le détachement est une forme d'appartenance qui semble se développer à la faveur du «nouvel esprit du capitalisme» déjà évoqué. Ce qui fait sens est réellement ce que l'on fait et ce que l'on est maintenant. Les lieux sont des occasions-supports pour tisser des liens, et les liens sont des points d'appui pour s'approprier ou fréquenter de nouveaux lieux. L'engagement de la personne se fait de façon à la fois plus intense mais plus réversible. On appartient aujourd'hui complètement à une communauté de projet, d'activité, de style de vie..., que l'on quittera bientôt. On peut parler ici d'une appartenance labile, en ce qu'elle est définie par l'inscription dans le temps présent. L'individu vit alors dans un présent permanent. Cette ouverture du présent peut, selon les personnes, être source d'incertitude ou d'opportunités. Les figures qui l'incarnent atteignent un haut niveau de détachement, de flexibi- 
lité, d'adaptabilité dans leurs rapports aux lieux et aux liens. Pour autant, leurs profils ne sont pas toujours ceux d'un dirigeant de firme multinationale. On y trouve au contraire toutes les palettes de la hiérarchie sociale. Ainsi, on peut évoquer ces jeunes saisonniers ou intérimaires circulant dans l'espace idéalisé de la liberté et du mouvement, dont le détachement vis-à-vis de leur entreprise ou du travail n'est que le pendant d'une socialisation professionnelle par la flexibilité.

Pour d'autres, le rapport au lieu est avant tout stratégique : qu'il soit vu comme le lieu idéal pour établir un pied-à-terre, parce qu'il offre l'accès à un paysage et à une nature préservés, ou le lieu d'une activité rémunératrice, il n'en reste pas moins pour les uns et les autres un lieu substituable que l'on quittera si d'autres opportunités se présentent.

\section{Enjeux sociaux et territoriaux des formes d'appartenance}

La diversité des formes d'appartenance laisse entrevoir la gageure que constitue le «vivre ensemble localisé » en milieu rural et les inégalités qui peuvent exister entre les habitants d'un lieu selon que celui-ci s'apparente pour eux à un choix de vie, à un arbitrage économique ou encore à un héritage non négociable.

Le premier enjeu est celui de la cohabitation. Contrairement aux villes où l'anonymat est une situation normale et parfois recherchée pour ses effets protecteurs, les campagnes ont été et demeurent dans les imaginaires le lieu de l'interconnaissance : cela explique que tant de personnes y convergent en croyant y retrouver une certaine convivialité et sociabilité villageoises. Mais l'interconnaissance au sens précis du terme (chacun connaît chacun sous tous les angles de sa vie sociale et individuelle, la régulation sociale s'effectuant directement par cet autocontrôle) a disparu dans les faits. Le problème n'est donc plus tant de se supporter durablement dans un contexte d'interconnaissance et d'enracinement, mais de se « reconnaitre » quand le voisin se présente de plus en plus sous les traits de l'étranger. La mobilité résidentielle, la segmentation des espaces de vie,

66 Avoir des liens ici et être allé voir ailleurs assure ainsi une double légitimité d'autochtone et d'étranger auprès des diverses catégories d'babitants. la diversité des formes d'inscription professionnelle et sociale rendent plus fragile la cohésion sociale locale.

Le récit commun entre habitants est ce qui fait défaut dans les situations conflictuelles qui rythment la vie des villages. Le retrait des services publics des zones rurales - l'école notamment, haut lieu de rencontre et d'insertion locale - affaiblit encore la possibilité de s'inscrire dans des lieux collectifs et une histoire commune. Le rôle de médiation des maires devient de plus en plus important, alors même que leur fonction ne les y prépare guère. Plus que des compétences, c'est souvent un parcours qui prédestine certains à 
assumer ce rôle délicat avec succès. Avoir des liens ici et être allé voir ailleurs assure ainsi une double légitimité d'autochtone et d'étranger auprès des diverses catégories d'habitants.

Le second enjeu concerne la place des uns et des autres dans l'espace géographique, autrement dit les questions de ségrégation sociale. Les inégalités décrites plus haut, en termes de capacité à maîtriser la mobilité, à choisir ses ancrages, à être partie prenante du lieu où l'on vit, montrent la fragilité des attachés et le pouvoir des autres catégories d'habitants sur l'espace. La relégation de territoires ruraux entiers (par enclavement, dévitalisation économique ou démographique, départ des services publics) est relativement présente dans les esprits (Berthod-Wusmer et al., 2009). La situation de relégation des couches populaires en dehors des villages ou régions fortement investis par des catégories plus fortunées est sans doute moins connue ; le coût du logement devient par exemple inabordable dans les régions touristiques et frontalières (Saddier, 2005). La difficulté de certains jeunes à se maintenir dans les villages soumis à la flambée immobilière en est un exemple, comme celle qu'ils ont, au contraire, à quitter leurs attaches dans des zones sinistrées par le chômage ou enclavées. La prise en compte des formes d'appartenance conduit à souligner l'importance de réelles politiques publiques (d'emploi, du logement, de la formation, du foncier...) qui garantissent le droit à la mobilité et à l'ancrage face aux logiques concurrentielles et de marché qui, de plus en plus, organisent le devenir des territoires et de leur population. Cependant, des initiatives locales se font jour et rappellent que le milieu rural est aussi un laboratoire d'alternatives, comme l'illustre le cas de Cobonne, un village rural en voie de périurbanisation (Drôme). La mairie a pris le parti de construire, avec l'aide d'un bailleur social, un lotissement à vocation sociale pour lutter contre le risque d'éviction des plus pauvres face à la hausse des prix de l'immobilier qui gagnait ce petit écrin de verdure à proximité de Valence.

Dans le même temps, un collectif d'habitants s'est constitué avec le projet de retisser des liens entre les différentes parties du village, dont le repeuplement s'est fait selon une logique de forte segmentation sociale entre les hameaux. L'association de médiation culturelle qui a porté l'opération a ainsi fait venir une compagnie artistique. La construction d'un bar à côté du local à poubelles - haut lieu de mixité puisque fréquenté par tous les habitants -, la circulation d'une malle médiathèque transportée par les enfants du village - ambassadeurs efficaces lorsqu'il s'agit de se faire ouvrir les portes - et des chantiers collectifs ont ainsi émaillé la vie de la commune et redonné un sens collectif au fait de vivre à la campagne. Cette dernière redeviendrait-elle finalement un foyer d'utopie politique, où l'attachement à des lieux et l'engagement dans des liens seraient à nouveau désirables et possibles? 
1 - Le capital d'autochtonie est la ressource symbolique, liée au fait d'être né ici, d'y avoir des parents, une lignée, dont peuvent jouir les classes populaires, peu pourvues en capital économique et culturel, pour s'insérer localement dans l'emploi (réputation, savoir-faire spécifique) et dans les activités sociales et culturelles locales (chasse, foot, sapeurs-pompiers). Ce capital est en crise lorsqu'il ne garantit plus l'accès à l'emploi local (du fait des délocalisations ou des formes de recrutement), à l'autonomie (pouvoir se loger, fonder une famille) ni même la reproduction sociale de son groupe.

\section{Bibliographie}

- Berthod-Wusmer M., Ollivier R., Raymond M., Villers S. et Fabre D., 2009, «Pauvreté, précarité, solidarité en milieu rural », rapport n 1883 de l'Inspection générale des affaires sociales (Igas) et du Conseil général de I'alimentation, de I'agriculture et des espace ruraux (CGAAER), disponible sur Internet.

- Boltanski L. et Chiapello È., 1999, Le nouvel esprit du capitalisme, Paris, Gallimard, coll. « Essais ».

- Kaufmann V., 2005, "Mobilités et réversibilités : vers des sociétés plus fluides?", Cahiers internationaux de sociologie, vol. CXVIII, p. 119-136.

- Mendras H., 1992, La fin des paysans, Arles, Actes Sud, (1 ${ }^{\text {re }}$ éd. 1977), coll. «Babel».

- Rénahy N., 2005, Les Gars du coin, enquête sur une jeunesse rurale, Paris, La Découverte, coll. « Textes à l'appui ».

- Saddier M., 2005, « Foncier-logement : les territoires touristiques et frontaliers sous haute pression », rapport au Premier ministre, Paris, La Documentation française.

- Sencébé Y., 2008, " Déclin(aisons) de l'appartenance dans les territoires de I'individualisme et de la mobilité », in Guérin-Pace F. et Filippova E. (dir.), Ces lieux qui nous habitent, La Tour d'Aigues, éd. de l'Aube, p. 37-50. 